Probability, Networks and Algorithms 
CWI is the National Research Institute for Mathematics and Computer Science. It is sponsored by the Netherlands Organization for Scientific Research (NWO).

CWI is a founding member of ERCIM, the European Research Consortium for Informatics and Mathematics.

CWI's research has a theme-oriented structure and is grouped into four clusters. Listed below are the names of the clusters and in parentheses their acronyms.

\section{Probability, Networks and Algorithms (PNA)}

Software Engineering (SEN)

Modelling, Analysis and Simulation (MAS)

Information Systems (INS)

Copyright (C) 2001, Stichting Centrum voor Wiskunde en Informatica

P.O. Box 94079, 1090 GB Amsterdam (NL)

Kruislaan 413, 1098 SJ Amsterdam (NL)

Telephone +31205929333

Telefax +31205924199

ISSN 1386-3711 


\title{
A Frequency Domain Approach to some Results on Fractional Brownian Motion
}

\author{
K. Dzhaparidze and J.A. Ferreira \\ $C W I$ \\ P.O. Box 94079, 1090 GB Amsterdam, The Netherlands \\ kacha@cwi.nl, jose.ferreira@cwi.nl
}

\begin{abstract}
Let $X$ be a fractional Brownian motion. It is known that $M_{t}=\int m_{t} d X, t \geq 0$, where $m_{t}$ is a certain kernel, defines a martingale $M$, and also that $X$ can be represented by $X_{t}=\int x_{t} d M, t>0$, for some kernel $x_{t}$. We derive these results by using the spectral representation of the covariance function of $X$. A formula for the covariance between $X$ and $M$ is also given.

2000 Mathematics Subject Classification: 60G15, $60 \mathrm{G} 18$.

Keywords and Phrases: Fractional Brownian motion, integral transforms, spectral representation.

Note: Work carried out under the project PNA3.3, 'Stochastic Processes and Applications'. The work of the second author was funded by The Fifth Framework Programme of the European Commission through the Dynstoch research network.
\end{abstract}

\section{Introduction}

A process $X=\left(X_{t}\right)_{t \geq 0}$ is said to be a fractional Brownian motion with self-similarity index $H \in$ $(0,1)$ if it is a continuous, centred gaussian process with $X_{0}=0$ and $E\left[\left(X_{t}-X_{s}\right)^{2}\right]=(t-s)^{2 H}$, $s, t \geq 0$. These specifications imply that $X$ has stationary increments and covariance function given by

$$
b(s, t)=\frac{1}{2}\left(t^{2 H}+s^{2 H}-|t-s|^{2 H}\right), \quad s, t \geq 0,
$$

from which it follows that $X$ is also a self-similar process with exponent $H$; that is, $X$ satisfies $\left(X_{\alpha t}\right)_{t \geq 0} \stackrel{d}{=}\left(\alpha^{H} X_{t}\right)_{t \geq 0}$ for all $\alpha>0$.

For $H=\frac{1}{2}$ fractional Brownian motion is a standard Brownian motion, and for $H \neq \frac{1}{2}$ it is not a semimartingale (Lipster and Shiryayev (1989), p. 300). In fact, one can check that the quadratic variation of $X$ is infinite if $H \in(0,1 / 2)$ and zero if $H \in(1 / 2,1)$.

The parameter $H$ is also called Hurst index. For brevity we sometimes use the shorthand notation $f B m(H)$ to refer to a fractional Brownian motion indexed by $H$.

The study of $f B m(H)$ goes back to Kolmogorov (1940), who showed in particular that (1.1) defines a covariance function if and only if $H \in(0,1]$ (the case $H=1$ corresponds to an a.s. linear random function). Mandelbrot and van Ness (1968) have given the name to the process, studied some of its properties, and suggested its practical use in connection with certain empirical studies of hydrological data by H.E. Hurst, from which the index $H$ derives its name. 
More recently, Barton and Poor (1988), Feyel and de La Pradelle (1996), Decreusefond and Üstünel (1999) and Norros et al. (1999), have rediscovered independently some of the results obtained in the 60's by Molchan (1969) and Molchan and Golosov (1969). In particular, these authors have found that the process $M$ defined by

$$
M_{t}=\int_{0}^{t} m_{t}(s) d X_{s}, \quad t \geq 0
$$

where

$$
m_{t}(s)=\frac{1}{2 H B\left(H+\frac{1}{2}, \frac{3}{2}-H\right)} s^{\frac{1}{2}-H}(t-s)^{\frac{1}{2}-H} 1_{(0, t)}(s), \quad s \geq 0,
$$

is a gaussian martingale with variance function $V$ given by

$$
V_{t} \equiv E M_{t}^{2}=\left(\frac{C_{H}}{2 H}\right)^{2} \frac{t^{2-2 H}}{2-2 H}, \quad t \geq 0, \quad \text { with } \quad C_{H}^{2} \equiv \frac{2 H \Gamma\left(\frac{3}{2}-H\right)}{\Gamma\left(H+\frac{1}{2}\right) \Gamma(2-2 H)} .
$$

The process $M$, which Norros et al. (1999) call the fundamental martingale, is easily seen to be related to a standard Brownian motion $W$ by the inverse relationships

$$
W_{t}=\frac{2 H}{C_{H}} \int_{0}^{t} s^{H-\frac{1}{2}} d M_{s}, \quad M_{t}=\frac{C_{H}}{2 H} \int_{0}^{t} s^{\frac{1}{2}-H} d W_{s}, \quad t \geq 0 .
$$

Among several related results and applications of (1.2), those authors have obtained a representation of $X$ as an integral with respect to $M$, namely

$$
X_{t}=2 H \int_{0}^{t} x_{t}(s) d M_{s}, \quad t \geq 0,
$$

where

$$
x_{t}(s)=\left\{t^{H-\frac{1}{2}}(t-s)^{H-\frac{1}{2}}-\int_{s}^{t}(u-s)^{H-\frac{1}{2}} d u^{H-\frac{1}{2}}\right\} 1_{(0, t)}(s), \quad s \geq 0 .
$$

This relation is the inverse of (1.2), and by virtue of (1.5) it is equivalent to

$$
X_{t}=C_{H} \int_{0}^{t} x_{t}(s) s^{\frac{1}{2}-H} d W_{s}, \quad t \geq 0,
$$

a representation of $f B m(H)$ in terms of a standard Brownian motion.

These representations hold not only in m.s. (mean square) but also a.s.; see Section 2.2 of Norros et al. (1999).

There are now relatively simple ways of proving (1.2) and (1.6). Norros et al. (1999), for instance, provide an elementary and self-contained presentation. Nuzman and Poor (2000) use Lamperti's transformation to map $f B m(H)$ into a stationary process and to translate classical results of the theory of stationary processes in terms of $f B m(H)$, and their approach, which uses the self-similarity property of $f B m(H)$, yields (1.2) and (1.6) as well as several prediction formulae in a rather elegant and efficient way. 
In spite of this, it seems of some interest to look at these results from the yet unexplored point of view of processes with stationary increments. In Section 2 of this paper we derive (1.2) and (1.6) from a 'frequency domain' point of view by taking advantage of the spectral representation of the covariance function of $f B m(H)$, a special case of the spectral representation for processes with stationary increments. Besides shedding new light on known results, this approach yields an interesting expression for the covariance between $X$ and $M$, proved in Section 3 , which might be useful for studying more complicated processes defined in terms of fractional Brownian motion.

Our approach relies on the theory of special functions (Bessel and hypergeometric functions). While this exhibits interesting connections, it makes our presentation less self-contained. To compensate for this shortcoming we have included an appendix summarizing those definitions and basic facts from special functions used in the proofs.

\section{Integral transforms: a spectral analysis approach}

The following spectral representation of the covariance function (1.1) is well-known from the theory of processes with stationary increments (e.g. Yaglom (1987), p. 407):

Proposition 2.1. The covariance function (1.1) is harmonizable in the sense that

$$
b(s, t)=\int_{-\infty}^{\infty} \overline{\hat{1}_{s}(\lambda)} \hat{1}_{t}(\lambda) d G(\lambda), \quad s, t \geq 0
$$

where $\hat{1}_{t}(\lambda)=\int_{-\infty}^{\infty} 1_{t}(s) e^{i \lambda s} d s=\frac{e^{i \lambda t}-1}{i \lambda}$ is the Fourier transform ${ }^{1}$ of the indicator function $1_{t}:=1_{(0, t)}$, and

$$
G(\lambda)=\frac{\left(C_{H} \Gamma\left(H+\frac{1}{2}\right)\right)^{2}}{2 \pi} \frac{\lambda^{2-2 H}}{2-2 H} \quad \text { for } \quad \lambda \geq 0, \quad G(\lambda)=-G(-\lambda) \quad \text { for } \quad \lambda<0 .
$$

For $H \in(1 / 2,1)$ the imaginary part of the integral in $(2.1)$ is not defined; in that case it is interpreted as zero. $G$ is called the spectral distribution function of $X$.

To verify (2.1) one writes out the integrand on the right-hand side and uses the fact that $\int_{0}^{\infty}(1-\cos x) x^{-2 H-1} d x=-\Gamma(-2 H) \cos (\pi H)$ (e.g. Gradshteyn and Rizhik (1980), formula 3.823) together with the identities (A.1) for the gamma function given in the Appendix.

Note that the spectral distribution function of $X$ and the variance function of $M$ are related by $G(t)=\left(2 H \Gamma\left(H+\frac{1}{2}\right) / \sqrt{2 \pi}\right)^{2} V_{t}, t \geq 0$. Thus Proposition 2.1 implies in particular the m.s. representation

$$
X_{t}=\frac{2 H \Gamma\left(H+\frac{1}{2}\right)}{\sqrt{2 \pi}} \int_{-\infty}^{\infty} \hat{1}_{t}(\lambda) d \tilde{M}_{\lambda}, \quad t \geq 0,
$$

where $\tilde{M}$ is a gaussian process with independent increments on the whole real line with the same variance function as $M$. This is equivalent to Hunt's (1951) frequency representation of $f B m(H)$ in terms of a standard Brownian motion; see also Samorodnitsky and Taqqu (1994).

\footnotetext{
${ }^{1}$ We define the Fourier transform of $f$ by $\hat{f}(\lambda)=\int e^{i \lambda x} f(x) d x, \lambda \in \mathbb{R}$.
} 
In order to formalize integration with respect to $f B m(H)$, let us denote by $\mathcal{I}_{\mathcal{H}}$ the set of functions in $L^{2}\left(\mathbb{R}_{+}\right)$whose Fourier-Plancherel transforms are square integrable on $\mathbb{R}$ with respect to $G$, and define an inner product on $\mathcal{I}_{\mathcal{H}}$ by

$$
\ll f, g \gg_{G}=\int_{-\infty}^{\infty} \Re\{\overline{\hat{f}(\lambda)} \hat{g}(\lambda)\} d G(\lambda) .
$$

By Proposition 2.1,

$$
\ll 1_{s}, 1_{t} \gg_{G}=E X_{s} X_{t},
$$

so the mapping $\sum_{k=1}^{n} c_{k} 1_{t_{k}} \longrightarrow \sum_{k=1}^{n} c_{k} X_{t_{k}}, c_{k} \in \mathbb{R}$, defines an isometry from the set of simple functions on $\mathbb{R}_{+}$onto the set of linear combinations of $X_{t}, t \geq 0$. Since the simple functions are dense in $\mathcal{I}_{\mathcal{H}}$, this mapping extends to an isometry from $\overline{\mathcal{I}_{\mathcal{H}}}$ (the closure of $\mathcal{I}_{\mathcal{H}}$ ) onto the gaussian Hilbert space generated by $X$, and therefore the integral $\int f d X$ is defined for $f \in \overline{\mathcal{I}_{\mathcal{H}}}$ as the image of $f$ under this isometry. Moreover, $E \int f d X=0, E\left[\left(\int f d X\right)^{2}\right]=\|f\|_{G}^{2}$, and $E \int f d X \int g d X=\ll f, g \gg_{G}, f, g \in \overline{\mathcal{I}_{\mathcal{H}}}$.

Pipiras and Taqqu (2000) have studied the problem of characterizing the class of functions $f$ for which $\int f d X$ can be defined in m.s., and showed in particular that $\mathcal{I}_{\mathcal{H}}$ is not a complete subspace of $L^{2}\left(\mathbb{R}_{+}\right)$for $H \neq 1 / 2$, so that $\mathcal{I}_{\mathcal{H}} \neq \overline{\mathcal{I}_{\mathcal{H}}}$ unless $H=1 / 2$. In what follows, however, the integrands considered are in $\mathcal{I}_{\mathcal{H}}$.

Our objective is to prove that (1.2) defines a martingale, and that the representations (1.6) and (1.8) hold in m.s. We begin by deriving a representation of $\hat{m}_{t}$, the Fourier transform of the kernel $m_{t}$ defined by (1.3), in terms of the Bessel function of order $1-H$; see A.2 in the Appendix for the definition of the Bessel function.

Proposition 2.2. For $\lambda \in \mathbb{R}, t \geq 0$,

$$
\hat{m}_{t}(\lambda)=\frac{\sqrt{\pi}}{2 H \Gamma\left(H+\frac{1}{2}\right)}\left(\frac{t}{\lambda}\right)^{1-H} e^{i \lambda t / 2} J_{1-H}\left(\frac{\lambda t}{2}\right) ;
$$

in particular,

$$
\hat{m}_{t}(0)=\left(\frac{C_{H}}{2 H}\right)^{2} \frac{t^{2-2 H}}{2-2 H}=V_{t}, \quad t \geq 0 .
$$

The representation (2.2) is in fact well-known; see for instance Samko et al. (1993), formula 10 in Table 9.1, or Gradshteyn and Rizhik (1980), formula 3.383(2). For completeness, however, we provide a simple proof based on the integral representation of Bessel functions (formula (A.4) in A.2).

Proof. By definition,

$$
2 H \Gamma\left(H+\frac{1}{2}\right) \Gamma\left(\frac{3}{2}-H\right) \hat{m}_{t}(\lambda)=\int_{0}^{t} s^{\frac{1}{2}-H}(t-s)^{\frac{1}{2}-H} e^{i \lambda s} d s .
$$

Multiply both sides of this equation by $e^{-i \lambda t / 2}$ and make the change of variable $u=\frac{t}{2}-s$ to get

$$
2 H \Gamma\left(H+\frac{1}{2}\right) \Gamma\left(\frac{3}{2}-H\right) e^{-i \lambda t / 2} \hat{m}_{t}(\lambda)=\int_{-t / 2}^{t / 2} e^{-i \lambda u}\left[\left(\frac{t}{2}\right)^{2}-u^{2}\right]^{\frac{1}{2}-H} d u .
$$


Since the integral on the right equals

$$
\int_{-t / 2}^{t / 2} \cos (\lambda u)\left[\left(\frac{t}{2}\right)^{2}-u^{2}\right]^{\frac{1}{2}-H} d u=\left(\frac{t}{2}\right)^{2-2 H} \int_{-1}^{1} \cos \left(\frac{\lambda t}{2} s\right)\left(1-s^{2}\right)^{\frac{1}{2}-H} d s
$$

(2.2) follows from the Poisson formula (A.4) with $\nu=1-H$ and $z=\lambda t / 2$. To get $\hat{m}_{t}(0)$ from (2.2) one uses the definitions of $J_{1-H}$ and $V$, and the second identity in (A.1).

Formula (2.3) below states that

$$
E M_{s} M_{t}=\ll m_{s}, m_{t} \gg_{G}=V_{s \wedge t}=\hat{m}_{s \wedge t}(0),
$$

which shows that the process $M$ in (1.2) has uncorrelated increments and hence is a martingale; cf. Theorem 3.1 of Norros et al. (1999).

Theorem 2.3. For $s, t \geq 0$,

$$
\ll m_{s}, m_{t} \gg_{G} \equiv \int_{-\infty}^{\infty} \overline{\hat{m}_{s}(\lambda)} \hat{m}_{t}(\lambda) d G(\lambda)=\hat{m}_{s \wedge t}(0) .
$$

Proof. The proof relies on the formula

$$
\int_{0}^{\infty} \frac{1}{x} \cos (a x) J_{\nu}(b x) J_{\nu}(c x) d x=\frac{1}{2 \nu}\left(\frac{b}{c}\right)^{\nu}
$$

which is valid for $\nu>0$ and $a \in[0, c-b], c \geq a+b$. This is a special case of a general formula derived by Bailey (1934) for a class of integrals involving products of Bessel functions; see Subsection A.5 in the Appendix.

In view of the representation $(2.2)$, we have

$$
\int_{-\infty}^{\infty} \overline{\hat{m}_{s}(\lambda)} \hat{m}_{t}(\lambda) d G(\lambda)=\frac{\pi(s t)^{1-H}}{\left(2 H \Gamma\left(H+\frac{1}{2}\right)\right)^{2}} \int_{-\infty}^{\infty} \lambda^{2 H-2} e^{i \lambda(t-s) / 2} J_{1-H}\left(\frac{\lambda s}{2}\right) J_{1-H}\left(\frac{\lambda t}{2}\right) d G(\lambda) .
$$

Therefore, using the property $J_{1-H}(-x)=(-1)^{1-H} J_{1-H}(x)$ (which follows directly from the definition of the Bessel function) and the symmetry of $G$, we see that what we need to show is that, for $s, t \geq 0$,

$$
\frac{2 \pi(s t)^{1-H}}{\left(2 H \Gamma\left(H+\frac{1}{2}\right)\right)^{2}} \int_{0}^{\infty} \lambda^{2 H-2} \cos \left(\frac{\lambda(t-s)}{2}\right) J_{1-H}\left(\frac{\lambda s}{2}\right) J_{1-H}\left(\frac{\lambda t}{2}\right) d G(\lambda)=V_{s \wedge t} .
$$

Suppose $t \geq s$. With $\nu=1-H, a=(t-s) / 2, b=s / 2$ and $c=t / 2$, formula (2.4) reads

$$
\int_{0}^{\infty} \lambda^{2 H-2} \cos \left(\frac{\lambda(t-s)}{2}\right) J_{1-H}\left(\frac{\lambda t}{2}\right) J_{1-H}\left(\frac{\lambda s}{2}\right) d G(\lambda)=\frac{\left(C_{H} \Gamma\left(H+\frac{1}{2}\right)\right)^{2}}{2 \pi(2-2 H)}\left(\frac{s}{t}\right)^{1-H},
$$

which is precisely (2.5). As the same argument applies with $s$ and $t$ interchanged, this proves (2.3). 
The m.s. representations of fractional Brownian motion in (1.6) and (1.8) are consequences of the following formula for the covariance function (1.1); cf. Theorem 5.2 of Norros et al. (1999).

Theorem 2.4. The covariance function (1.1) has the representation

$$
b(s, t)=(2 H)^{2} \int_{0}^{s \wedge t} x_{s}(u) x_{t}(u) d V_{u}, \quad s, t \geq 0,
$$

where $x_{t}$ is defined by (1.3) and $V$ by (1.4).

To prove Theorem 2.4 we shall need two lemmas.

Lemma 2.5. We have

$$
\int_{-\infty}^{+\infty} \overline{\int_{0}^{s} y_{s}(u) d_{u} \hat{m}_{u}(\lambda)} \int_{0}^{t} z_{t}(v) d_{v} \hat{m}_{v}(\lambda) d G(\lambda)=\int_{0}^{s \wedge t} y_{s}(u) z_{t}(u) d_{u} \hat{m}_{u}(0)
$$

for all non-negative kernels $y_{s}, z_{t}$ that are continuously differentiable on their supports $(0, s)$ and $(0, t)$.

Proof. Since both $u \rightarrow \hat{m}_{u}(0)$ and $\lambda \rightarrow \hat{m}_{u}(\lambda)$ are of bounded variation, it is enough to prove the result for $y_{s}$ and $z_{t}$ continuous on $[0, s]$ and $[0, t]$, because if the functions are discontinuous at the endpoints of their intervals of support, or even unbounded, we can approximate them monotonically from below by continuous functions and then pass to the limit in (2.7). In this case integration by parts gives $\int_{0}^{t} y_{s}(u) d_{u} \hat{m}_{u}(\lambda)=-\int_{0}^{t} \hat{m}_{u}(\lambda) y_{s}(d u)$ and $\int_{0}^{t} z_{t}(v) d_{v} \hat{m}_{v}(\lambda)$ $=-\int_{0}^{t} \hat{m}_{v}(\lambda) z_{t}(d v)$, hence

$$
\overline{\int_{0}^{s} y_{s}(u) d_{u} \hat{m}_{u}(\lambda)} \int_{0}^{t} z_{t}(v) d_{v} \hat{m}_{v}(\lambda)=\int_{0}^{t} \int_{0}^{s} \hat{m}_{u}(\lambda) \overline{\hat{m}_{v}(\lambda)} y_{s}(d u) z_{t}(d v) .
$$

Integrating with respect to $G$ and using (2.3), we get, for $s \leq t$,

$$
\begin{gathered}
\int_{-\infty}^{+\infty} \int_{0}^{t} z_{t}(v) d_{v} \hat{m}_{v}(\lambda) \overline{\int_{0}^{s} y_{s}(u) d_{u} \hat{m}_{u}(\lambda)} d G(\lambda)=\left(\frac{C_{H}}{2 H}\right)^{2} \frac{1}{2-2 H} \times \\
\left(\int_{0}^{s} \int_{v}^{s} v^{2-2 H} y_{s}(d u) z_{t}(d v)+\int_{0}^{s} \int_{u}^{s} u^{2-2 H} z_{t}(d v) y_{s}(d u)+\int_{0}^{s} \int_{s}^{t} u^{2-2 H} z_{t}(d v) y_{s}(d u)\right)= \\
-\left(\frac{C_{H}}{2 H}\right)^{2} \frac{1}{2-2 H}\left(\int_{0}^{s} v^{2-2 H} y_{s}(v) z_{t}(d v)+\int_{0}^{s} u^{2-2 H} z_{t}(u) y_{s}(d u)\right)= \\
-\left(\frac{C_{H}}{2 H}\right)^{2} \frac{1}{2-2 H} \int_{0}^{s} v^{2-2 H} d_{u}\left(y_{s}(u) z_{t}(u)\right)=\int_{0}^{s} y_{s}(u) z_{t}(u) d_{u} \hat{m}_{u}(0),
\end{gathered}
$$

proving (2.7).

Lemma 2.6. For $t \geq 0$ and $\lambda \in \mathbb{R}$,

$$
\hat{1}_{t}(\lambda)=2 H \int_{0}^{t} x_{t}(s) d \hat{m}_{s}(\lambda)
$$


Proof. We first note that if $H \in(0,1 / 2)$ then

$$
2 H \int_{0}^{t}(t-s)^{H-\frac{1}{2}} d m_{s}(x)=x^{\frac{1}{2}-H} 1_{t}(x), \quad x>0,
$$

while if $H \in(1 / 2,1)$

$$
2 H \int_{0}^{t} m_{s}(x) d_{s}(t-s)^{H-\frac{1}{2}}=-x^{\frac{1}{2}-H} 1_{t}(x), \quad x>0 .
$$

These follow from the definitions of $m_{s}$ and the beta function.

Taking Fourier transforms on both sides of (2.9) gives us

$$
2 H \int_{0}^{t}(t-s)^{H-\frac{1}{2}} d \hat{m}_{s}(\lambda)=\int_{0}^{t} x^{\frac{1}{2}-H} e^{i x \lambda} d x .
$$

Doing the same with (2.10) and then integrating by parts, we see that (2.11) holds for all $H \in(0,1)$.

On the other hand, integration by parts and Fubini's theorem yield

$$
\begin{aligned}
\int_{0}^{t} u^{H-\frac{1}{2}} d \int_{0}^{u}(u-s)^{H-\frac{1}{2}} d \hat{m}_{s}(\lambda) & =t^{H-\frac{1}{2}} \int_{0}^{t}(t-s)^{H-\frac{1}{2}} d \hat{m}_{s}(\lambda)-\int_{0}^{t} \int_{0}^{u}(u-s)^{H-\frac{1}{2}} d \hat{m}_{s}(\lambda) d u^{H-\frac{1}{2}} \\
& =\int_{0}^{t}\left[t^{H-\frac{1}{2}}(t-s)^{H-\frac{1}{2}}-\int_{s}^{t}(u-s)^{H-\frac{1}{2}} d u^{H-\frac{1}{2}}\right] d \hat{m}_{s}(\lambda) \\
& =\int_{0}^{t} x_{t}(s) d \hat{m}_{s}(\lambda),
\end{aligned}
$$

so by $(2.11)$

$$
2 H \int_{0}^{t} x_{t}(s) d \hat{m}_{s}(\lambda)=\int_{0}^{t} u^{H-\frac{1}{2}} d \int_{0}^{u} x^{\frac{1}{2}-H} e^{i x \lambda} d x=\hat{1}_{t}(\lambda)
$$

proving (2.8).

Proof of Theorem 2.4: Recalling $\hat{m}_{u}(0)=V_{u}, u \geq 0$, and using Proposition 2.1, formula (2.8) and Lemma 2.5, we see that

$$
b(s, t)=(2 H)^{2} \int_{-\infty}^{\infty} \overline{\int_{0}^{s} x_{s}(u) d \hat{m}_{u}(\lambda)} \int_{0}^{t} x_{t}(v) d \hat{m}_{v}(\lambda) d G(\lambda)=(2 H)^{2} \int_{0}^{s \wedge t} x_{s}(u) x_{t}(u) d V_{u},
$$

which is (2.6).

\section{Formula for the covariance between $X$ and $M$}

In this section we obtain an expression for $E X_{s} M_{t}=\ll 1_{s}, m_{t} \gg_{G}$ in terms of Gauss's hypergeometric function $F$, whose definition and integral representation are given in Section A.3 of the Appendix. (Cf. Norros et al. (1999), Proposition 3.2.) 
Proposition 3.1. For $s, t \geq 0$,

$$
\ll 1_{s}, m_{t} \gg_{G} \equiv \int_{-\infty}^{\infty} \overline{\hat{1}_{s}(\lambda)} \hat{m}_{t}(\lambda) d G(\lambda)=s \times\left\{\begin{array}{cc}
1 & \text { if } s \leq t \\
\Psi_{t / s} & \text { if } s>t,
\end{array}\right.
$$

where

$$
\Psi_{\theta}=\frac{1}{2}\left(\theta+\theta^{2-2 H} \frac{F\left(1-2 H, \frac{3}{2}-H ; 3-2 H ; \theta\right)}{F\left(1-2 H, \frac{3}{2}-H ; 3-2 H ; 1\right)}\right), \quad 0 \leq \theta \leq 1 .
$$

Proof. From the representation of $\hat{m}_{t}$, we have

$$
\begin{aligned}
\Re\left\{\hat{m}_{t}(x)\left(\frac{1-e^{-i x s}}{i x}\right)\right\} & =\frac{\sqrt{\pi}\left(\frac{t}{x}\right)^{1-H} J_{1-H}\left(\frac{x t}{2}\right)}{2 H \Gamma\left(H+\frac{1}{2}\right)}\left\{e^{i x t / 2}\left(\frac{1-e^{-i x s}}{i x}\right)\right\} \\
& =\frac{\sqrt{\pi}\left(\frac{t}{x}\right)^{1-H} J_{1-H}\left(\frac{x t}{2}\right)}{2 H \Gamma\left(H+\frac{1}{2}\right)}\left\{\frac{\sin \left(\frac{x t}{2}\right)+\sin \left(x\left(s-\frac{t}{2}\right)\right)}{x}\right\},
\end{aligned}
$$

so

$$
\begin{aligned}
\ll 1_{s}, m_{t} \gg_{G}= & \frac{C_{H}^{2} \Gamma\left(H+\frac{1}{2}\right) t^{1-H}}{2 H \sqrt{\pi}}\left\{\int_{0}^{\infty} \frac{\sin \left(\frac{x t}{2}\right) J_{1-H}\left(\frac{x t}{2}\right)}{x^{1+H}} d x+\right. \\
& \left.\int_{0}^{\infty} \frac{\sin \left(x\left(s-\frac{t}{2}\right)\right) J_{1-H}\left(\frac{x t}{2}\right)}{x^{1+H}} d x\right\} .
\end{aligned}
$$

Since $\sin \left(\frac{x t}{2}\right)+\sin \left(x\left(s-\frac{t}{2}\right)\right)=2 \sin \left(\frac{x s}{2}\right) \cos \left(\frac{x(t-s)}{2}\right)$, we also have the alternative form

$$
\ll 1_{s}, m_{t} \gg_{G}=\frac{C_{H}^{2} \Gamma\left(H+\frac{1}{2}\right) t^{1-H}}{H \sqrt{\pi}} \int_{0}^{\infty} \frac{\sin \left(\frac{x s}{2}\right) \cos \left(\frac{x(t-s)}{2}\right) J_{1-H}\left(\frac{x t}{2}\right)}{x^{1+H}} d x,
$$

which is a more convenient expression when $t \geq s \geq 0$. To evaluate the integral involved in (3.4) in this case we use the identity

$$
\int_{0}^{\infty} x^{\rho-2} \cos (a x) \sin (b x) J_{\rho}(c x) d x=2^{\rho-1} c^{-\rho} b \Gamma(\rho), \quad \rho \in(0,3 / 2), a \in[0, c-b],
$$

again a special case of Bailey's general formula quoted in A.5. Taking $\rho=1-H, a=(t-s) / 2$, $b=s / 2$ and $c=t / 2$ here, we get

$$
\int_{0}^{\infty} \frac{\sin \left(\frac{x s}{2}\right) \cos \left(\frac{x(t-s)}{2}\right) J_{1-H}\left(\frac{x t}{2}\right)}{x^{1+H}} d x=\frac{s t^{H-1} \Gamma(1-H)}{2^{2 H}},
$$

from which we see that (3.4) is

$$
\ll 1_{s}, m_{t} \gg_{G}=s \frac{C_{H}^{2} \Gamma\left(H+\frac{1}{2}\right) \Gamma(1-H)}{H \sqrt{\pi} 2^{2 H}}=s,
$$


the last equality following from an application of the second identity in (A.1).

To prove (3.1) in the case $s>t \geq 0$ we go back to (3.3). The two integrals we now need to compute are special cases of the Weber-Schafheitlin integrals, which are themselves particular cases of the integrals considered by Bailey, and for these we have again formulae available; see (A.12) and (A.13) in A.5.

Since $\sin \left(\frac{t x}{2}\right)=\frac{\sqrt{t x \pi}}{2} J_{1 / 2}\left(\frac{t x}{2}\right)$, we may apply (A.12) with $\nu=1 / 2, \lambda=\rho=1-H$ and $b=t / 2$ to get

$$
\begin{aligned}
\int_{0}^{\infty} \frac{\sin \left(\frac{x t}{2}\right) J_{1-H}\left(\frac{x t}{2}\right)}{x^{1+H}} d x & =\frac{\sqrt{t \pi}}{2} \int_{0}^{\infty} \frac{J_{1 / 2}\left(\frac{t}{2} x\right) J_{1-H}\left(\frac{t}{2} x\right)}{x^{\frac{1}{2}+H}} d x \\
& =\Gamma(1-H)\left(\frac{t}{4}\right)^{H} .
\end{aligned}
$$

Similarly, using (A.13) with $\rho=1 / 2, \lambda=\nu=1-H, b=t / 2$ and $c=s-t / 2$, we find

$$
\begin{aligned}
\int_{0}^{\infty} \frac{\sin \left(x\left(s-\frac{t}{2}\right)\right) J_{1-H}\left(\frac{x t}{2}\right)}{x^{1+H}} d x= & \sqrt{\frac{\left(s-\frac{t}{2}\right) \pi}{2}} \int_{0}^{\infty} \frac{J_{1 / 2}\left(\left(s-\frac{t}{2}\right) x\right) J_{1-H}\left(\frac{t}{2} x\right)}{x^{\frac{1}{2}+H}} d x \\
= & \frac{\sqrt{\pi}}{2^{H+1}(1-H) \Gamma\left(H+\frac{1}{2}\right)} \frac{\left(\frac{t}{2}\right)^{1-H}}{\left(s-\frac{t}{2}\right)^{1-2 H}} \times \\
& F\left(1-H, \frac{1}{2}-H ; 2-H ;\left(\frac{t / s}{2-t / s}\right)^{2}\right) .
\end{aligned}
$$

Inserting these expressions in the formula for $\ll 1_{s}, m_{t} \gg_{G}$ and simplifying some of the constant terms, we obtain

$$
\ll 1_{s}, m_{t} \gg_{G}=\frac{t}{2}+\frac{\sqrt{\pi} t\left(\frac{t / s}{2-t / s}\right)^{1-2 H}}{4 \Gamma(2-H) \Gamma\left(H+\frac{1}{2}\right)} F\left(1-H, \frac{1}{2}-H ; 2-H ;\left(\frac{t / s}{2-t / s}\right)^{2}\right) .
$$

It follows from (3.5) that $\ll 1_{s}, m_{t} \gg_{G}=s \ll 1_{1}, m_{t / s} \gg_{G}$ (alternatively, this can be seen directly by changing variables in the integral defining $\left.\ll 1_{s}, m_{t} \gg_{G}\right)$. Thus, it is enough to prove (3.1) with $s$ replaced by 1 and $t$ replaced by $\theta:=t / s \in[0,1)$, which is notationally more convenient.

An appropriate change of variables in the integral representation of $F$ shows that

$$
F\left(\frac{\alpha+1}{2}, \frac{\alpha}{2} ; \beta+\frac{1}{2} ;\left(\frac{\theta}{2-\theta}\right)^{2}\right)=\left(1-\frac{\theta}{2}\right)^{\alpha} F(\alpha, \beta ; 2 \beta ; \theta),
$$

an identity valid at least for $\theta \in[0,1), \alpha-\beta \notin \mathbb{Z}-\{0\}$, which fits precisely our situation if we choose $\alpha=1-2 H, \beta=3 / 2-H$ (cf. Gradshteyn and Rizhik (1980), p. 1043). Thus we have

$$
F\left(1-H, \frac{1}{2}-H ; 2-H ;\left(\frac{\theta}{2-\theta}\right)^{2}\right)=\left(1-\frac{\theta}{2}\right)^{1-2 H} F\left(1-2 H, \frac{3}{2}-H ; 3-2 H ; \theta\right),
$$


and therefore

$$
\ll 1_{1}, m_{\theta} \gg_{G}=\frac{\theta}{2}+\left(\frac{\theta}{2}\right)^{2-2 H} \frac{\sqrt{\pi}}{2} \frac{F\left(1-2 H, \frac{3}{2}-H ; 3-2 H ; \theta\right)}{\Gamma\left(H+\frac{1}{2}\right) \Gamma(2-H)}, \quad \theta \in[0,1) .
$$

Noting that $F\left(1-2 H, \frac{3}{2}-H ; 3-2 H ; 1\right)=2^{2-2 H} \Gamma\left(H+\frac{1}{2}\right) \Gamma(2-H) / \sqrt{\pi}$, we can finally write this as

$$
\ll 1_{1}, m_{\theta} \gg_{G}=\frac{\theta}{2}\left\{1+\theta^{1-2 H} \frac{F\left(1-2 H, \frac{3}{2}-H ; 3-2 H ; \theta\right)}{F\left(1-2 H, \frac{3}{2}-H ; 3-2 H ; 1\right)}\right\} .
$$

To get (3.1) for $\theta=t / s \geq 0$ recall $\ll 1_{s}, m_{t} \gg_{G}=s \ll 1_{1}, m_{\theta} \gg_{G}$.

Remark 3.2. Once the representation (1.6) and the fact that $M$ is a martingale have been established, it follows that $\ll 1_{s}, m_{t} \gg_{G}=E X_{s} M_{t}=E\left[\int_{0}^{s \wedge t} x_{s}(u) d M_{u} M_{t}\right]=2 H \int_{0}^{s \wedge t} x_{s}(u) d V_{u}$. Thus (3.1) with $s=1$ shows that $\Psi_{t}=\int_{0}^{t} x_{1}(v) d V_{v}, t \in[0,1]$, so that (3.2) defines a distribution function and

$$
x_{1}(t)=\frac{d \Psi_{t}}{d V_{t}} .
$$

Remark 3.3. Using the formula $\ll 1_{s}, m_{t} \gg_{G}=2 H \int_{0}^{s \wedge t} x_{s}(u) d V_{u}$, noted in the previous remark, we can derive an alternative formula for $\Psi$ in an elementary way:

$$
\Psi_{\theta}=\theta+\frac{\left\{(1-\theta) \int_{0}^{\theta} u^{1-2 H}(1-u)^{H-\frac{3}{2}} d u+\theta \int_{\theta}^{1} u^{-2 H}(1-u)^{H-\frac{1}{2}} d u\right\}}{B\left(H-\frac{1}{2}, 2-2 H\right)}, \quad 0 \leq \theta \leq 1 .
$$

To prove it, we need to show that the integral

$$
\int_{0}^{\theta} x_{1}(v) d V_{v}=\frac{C_{H}^{2}}{2 H} \int_{0}^{\theta}\left\{(1-v)^{H-\frac{1}{2}}-\int_{v}^{1}(u-v)^{H-\frac{1}{2}} d u^{H-\frac{1}{2}}\right\} v^{1-2 H} d v
$$

equals the expression for $\Psi_{\theta}$ in (3.6). Using integration by parts, we can write

$$
(1-v)^{H-\frac{1}{2}}-\int_{v}^{1}(u-v)^{H-\frac{1}{2}} d u^{H-\frac{1}{2}}=\int_{\theta}^{1} u^{H-\frac{1}{2}} d_{u}(u-v)^{H-\frac{1}{2}}-\int_{v}^{\theta}(u-v)^{H-\frac{1}{2}} d u^{H-\frac{1}{2}},
$$

so

$$
\begin{aligned}
\int_{0}^{\theta} x_{1}(v) d V_{v}= & \frac{C_{H}^{2}}{2 H}\left\{\theta^{H-\frac{1}{2}} \int_{0}^{\theta}(\theta-v)^{H-\frac{1}{2}} v^{1-2 H} d v+\int_{0}^{\theta} \int_{\theta}^{1} u^{H-\frac{1}{2}} d_{u}(u-v)^{H-\frac{1}{2}} v^{1-2 H} d v-\right. \\
& \left.\int_{0}^{\theta} \int_{v}^{\theta}(u-v)^{H-\frac{1}{2}} d u^{H-\frac{1}{2}} v^{1-2 H} d v\right\}
\end{aligned}
$$

The first integral in this expression can be computed as

$$
\theta^{H-\frac{1}{2}} \int_{0}^{\theta}(\theta-v)^{H-\frac{1}{2}} v^{1-2 H} d v=\theta B\left(H+\frac{1}{2}, 2-2 H\right) .
$$


Let us simplify the double integrals. Changing variables $(x=u / v$ and then $u=1 / x)$ and then interchanging the order of integration, we get successively

$$
\begin{aligned}
\int_{0}^{\theta} \int_{v}^{\theta}(u-v)^{H-\frac{1}{2}} d u^{H-\frac{1}{2}} v^{1-2 H} d v & =\int_{0}^{\theta} \int_{v}^{\theta}\left(\frac{u}{v}-1\right)^{H-\frac{1}{2}} d u\left(\frac{u}{v}\right)^{H-\frac{1}{2}} d v \\
& =\int_{0}^{\theta} \int_{1}^{\frac{\theta}{v}}(x-1)^{H-\frac{1}{2}} d x^{H-\frac{1}{2}} d v \\
& =-\frac{1}{2} \int_{0}^{\theta} \int_{\frac{v}{\theta}}^{1}(1-u)^{H-\frac{1}{2}} d u^{1-2 H} d v \\
& =-\frac{1}{2} \int_{0}^{1} \int_{0}^{\theta u} d v(1-u)^{H-\frac{1}{2}} d u^{1-2 H} \\
& =\theta\left(H-\frac{1}{2}\right) \int_{0}^{1}(1-u)^{H-\frac{1}{2}} u^{1-2 H} d u \\
& =\theta\left(H-\frac{1}{2}\right) B\left(H+\frac{1}{2}, 2-2 H\right)
\end{aligned}
$$

Similarly,

$$
\begin{aligned}
\int_{0}^{\theta} \int_{\theta}^{1} u^{H-\frac{1}{2}} d_{u}(u-v)^{H-\frac{1}{2}} v^{1-2 H} d v= & \int_{0}^{\theta} \int_{\theta}^{1}\left(\frac{u}{v}\right)^{H-\frac{1}{2}} d_{u}\left(\frac{u}{v}-1\right)^{H-\frac{1}{2}} d v \\
= & -\int_{0}^{\theta} \int_{v}^{\frac{v}{\theta}} u^{\frac{1}{2}-H} d\left(\frac{1-u}{u}\right)^{H-\frac{1}{2}} d v \\
= & -\int_{0}^{\theta} \int_{\theta u}^{u} d v u^{\frac{1}{2}-H} d\left(\frac{1-u}{u}\right)^{H-\frac{1}{2}} \\
& -\int_{0}^{\theta} \int_{\theta u}^{\theta} d v u^{\frac{1}{2}-H} d\left(\frac{1-u}{u}\right)^{H-\frac{1}{2}} \\
= & \left(H-\frac{1}{2}\right)\left\{(1-\theta) \int_{0}^{\theta} u^{1-2 H}(1-u)^{H-\frac{3}{2}} d u+\right. \\
& \left.\theta \int_{\theta}^{1} u^{-2 H}(1-u)^{H-\frac{1}{2}} d u\right\}
\end{aligned}
$$

Inserting these formulae in (3.7) and simplifying the constant terms we get $\int_{0}^{\theta} x_{1}(v) d V_{v}=\Psi_{\theta}$.

Note that this is a 'time domain' proof of (3.1) for $s>t$; the case $s \leq t$ was proved in Norros et al. (1999).

\section{A Appendix on Special Functions}

\section{A.1 The gamma function}

The gamma function is usually defined for all $x>0$ by means of the formula

$$
\Gamma(x)=\int_{0}^{\infty} e^{-t} t^{x-1} d t
$$


and following the well-known identity $\Gamma(x+1)=x \Gamma(x), x>0$, this definition can be extended recursively to $x \neq 0,-1,-2, \ldots$ by setting $\Gamma(x)=\Gamma(x+1) / x$. Apart from these definitions we shall need the fact that $\Gamma\left(\frac{1}{2}\right)=\sqrt{\pi}$ and the famous identities

$$
\Gamma(x) \Gamma(1-x)=\frac{\pi}{\sin (\pi x)}, \quad \sqrt{\pi} \Gamma(2 x)=2^{2 x-1} \Gamma(x) \Gamma\left(x+\frac{1}{2}\right), \quad x \neq 0,-1,-2, \ldots
$$

(e.g. Lebedev (1972), formulae (1.2.2) and (1.2.3)).

\section{A.2 The Bessel function}

The Bessel function of the first kind of order $\nu \neq-1,-2, \ldots$ is defined for all complex $|z|<\infty$ with $|\arg z|<\pi$ as the absolutely convergent sum

$$
J_{\nu}(z)=\sum_{k=0}^{\infty} \frac{(-1)^{k}(z / 2)^{\nu+2 k}}{\Gamma(k+1) \Gamma(\nu+k+1)} .
$$

As special cases we have

$$
J_{\frac{1}{2}}(z)=\sqrt{\frac{2}{\pi z}} \sin (z) \text { and } J_{-\frac{1}{2}}(z)=\sqrt{\frac{2}{\pi z}} \cos (z), \quad z \neq 0 .
$$

We shall need the following integral representation of the Bessel function, known as the Poisson formula: for $\nu>-1 / 2$,

$$
J_{\nu}(z)=\frac{\left(\frac{z}{2}\right)^{\nu}}{\Gamma\left(\frac{1}{2}\right) \Gamma\left(\nu+\frac{1}{2}\right)} \int_{-1}^{1} \cos (z x)\left(1-x^{2}\right)^{\nu-\frac{1}{2}} d x, \quad z \in \mathbb{C}
$$

see e.g. Lebedev (1972), formula (5.10.3), or Samko et al. (1993), formula (2.52).

It is also useful to know, especially for studying the convergence of integrals of Bessel functions, that $J_{\nu}(x)$ behaves, for large real $x$, approximately as a linear combination of $x^{-1 / 2} \cos x$ and $x^{-1 / 2} \sin x$. More precisely,

$$
J_{\nu}(x)=c_{\nu} x^{-1 / 2} \cos (x)+c_{\nu}^{\prime} x^{-1 / 2} \sin (x)+x^{-3 / 2} r(x),
$$

where $c_{\nu}$ and $c_{\nu}^{\prime}$ are constants and $r(x)$ is bounded as $x \rightarrow+\infty$; see for instance Lebedev (1972).

\section{A.3 The hypergeometric function}

The hypergeometric function with parameters $\alpha, \beta, \gamma$ is the power series defined for all complex $|z|<1$ by

$$
F(\alpha, \beta ; \gamma ; z)=\sum_{k=0}^{\infty} \frac{(\alpha)_{k}(\beta)_{k}}{(\gamma)_{k}} \frac{z^{k}}{k !},
$$

where as usual we write $(\alpha)_{0}=1,(\alpha)_{k}:=\alpha(\alpha+1) \cdots(\alpha+k), k \in \mathbb{N}$. For this definition to make sense we require that $\gamma \neq 0,-1,-2, \ldots$; further, for the series to be convergent we assume $\alpha+\beta-\gamma>1$. Observe that $F$ is symmetric with respect to the parameters $\alpha$ and $\beta$, and also 
that it is identically equal to 1 if $\alpha=0$ or $\beta=0$. See for instance Lebedev (1972), Section 9.1, for more information.

We shall need the integral representation

$$
F(\alpha, \beta ; \gamma ; z)=\frac{\Gamma(\gamma)}{\Gamma(\beta) \Gamma(\gamma-\beta)} \int_{0}^{1} x^{\beta-1}(1-x)^{\gamma-\beta-1}(1-x z)^{-\alpha} d x,
$$

which is valid for $|z|<1$ and $\gamma>\beta>0$. When $z=1$ the integral on the right is the beta integral $B(\beta, \gamma-\beta-\alpha)$, which can be computed in terms of gamma functions by the well-known identity $B(x, y)=\Gamma(x) \Gamma(y) / \Gamma(x+y), x, y>0$.

\section{A.4 A hypergeometric function of two variables}

The fourth hypergeometric function of two variables with parameters $\alpha, \beta, \gamma, \gamma^{\prime}$ is defined as the power series

$$
F_{4}\left(\alpha, \beta ; \gamma, \gamma^{\prime} ; x, y\right)=\sum_{m=0}^{\infty} \sum_{n=0}^{\infty} \frac{(\alpha)_{m+n}(\beta)_{m+n}}{(\gamma)_{m}\left(\gamma^{\prime}\right)_{n}} \frac{x^{m}}{m !} \frac{y^{n}}{n !}, \quad|\sqrt{x}|+|\sqrt{y}|<1,
$$

where $\gamma, \gamma^{\prime} \neq 0,-1,-2, \ldots$ (Gradshteyn and Rizhik (1980), Section 9.18). This is one of several generalizations of the hypergeometric function $F$ to a function of two variables. Note that $F_{4}$ reduces to $F$ when one of the variables is set to zero; for example, $F_{4}\left(\alpha, \beta ; \gamma, \gamma^{\prime} ; 0, y\right)=$ $F\left(\alpha, \beta ; \gamma^{\prime} ; y\right)$. Also, $F_{4}$ equals 1 if $\alpha=0$ or $\beta=0$.

\section{A.5 Some integrals involving Bessel functions}

Bailey (1934) derived the following formula for an integral involving the product of three Bessel functions (see also Gradshteyn and Rizhik (1980), Table 6.578). Let $a, b$ and $c$ be real positive numbers such that $c>a+b$. Then for $-(\mu+\nu+\rho)<\lambda<\frac{5}{2},-\mu,-\nu,-\rho \notin \mathbb{N}$, we have

$$
\begin{aligned}
\int_{0}^{\infty} x^{\lambda-1} J_{\mu}(a x) J_{\nu}(b x) J_{\rho}(c x) d x= & \frac{2^{\lambda-1} a^{\mu} b^{\nu} \Gamma(\beta)}{c^{\lambda+\mu+\nu} \Gamma(\mu+1) \Gamma(\nu+1) \Gamma(1-\alpha)} \times \\
& F_{4}\left(\alpha, \beta ; \mu+1, \nu+1 ;\left(\frac{a}{c}\right)^{2},\left(\frac{b}{c}\right)^{2}\right),
\end{aligned}
$$

where $\alpha:=\frac{1}{2}(\lambda+\mu+\nu-\rho)$ and $\beta:=\frac{1}{2}(\lambda+\mu+\nu+\rho)$.

The condition $c>a+b$ is necessary for $F_{4}$ to be defined (see (A.7)). It can be relaxed to $c \geq a+b$ in certain special instances, some of which will be discussed below. From (A.5) it can be seen that the integral in (A.8) is absolutely convergent if and only if $\mu+\nu+\rho<\lambda<3 / 2$.

If $\lambda=\rho-\mu-\nu$, then $\alpha=0$ and the factor $F_{4}$ reduces to unity, so Bailey's formula simplifies to

$$
\int_{0}^{\infty} x^{\rho-\mu-\nu-1} J_{\mu}(a x) J_{\nu}(b x) J_{\rho}(c x) d x=\frac{2^{\rho-\mu-\nu-1} a^{\mu} b^{\nu} \Gamma(\rho)}{c^{\rho} \Gamma(\mu+1) \Gamma(\nu+1)},
$$

$\rho>0, \rho-\mu-\nu<\frac{5}{2}$. 
Setting $\mu=-\frac{1}{2}$ in (A.9) and using the second identity in (A.3), we obtain

$$
\int_{0}^{\infty} x^{\rho-\nu-1} \cos (a x) J_{\nu}(b x) J_{\rho}(c x) d x=\frac{2^{\rho-\nu-1} b^{\nu} \Gamma(\rho)}{c^{\rho} \Gamma(\nu+1)}
$$

$0<\rho<2+\nu, a \in(0, c-b)$. By (A.3), it can be seen that if $\rho>0$ and $\rho-\nu<1$ then the integrand in this equation can be bounded above in absolute value by an integrable function; therefore, by the dominated convergence theorem we can let $a \rightarrow 0+$ or $a \rightarrow(c-b)-$ on the left-hand side without affecting the right-hand side, and it follows that formula (A.10) is valid for $0<\rho<1+\nu, a \in[0, c-b]$. This result is used in the proof of Theorem 2.3 in the form of equation (2.4), obtained from (A.10) with $\nu=\rho$.

Next, letting $\nu=\frac{1}{2}$ in (A.10) yields

$$
\int_{0}^{\infty} x^{\rho-2} \cos (a x) \sin (b x) J_{\rho}(c x) d x=2^{\rho-1} c^{-\rho} b \Gamma(\rho),
$$

again valid for all $a \in[0, c-b]$ if $0<\rho<3 / 2$, a result used in the proof of Proposition 3.1.

Bailey's result also contains formulae for the Weber-Schafheitlin integrals (Watson (1944), pp. 401, 403), namely

$$
\begin{aligned}
\int_{0}^{\infty} \frac{J_{\nu}(b x) J_{\rho}(b x)}{x^{\frac{3}{2}-\lambda}} d x= & \frac{2^{\lambda-\frac{3}{2}} b^{\frac{1}{2}-\lambda} \Gamma\left(\frac{1}{2}\left(\lambda+\nu+\rho-\frac{1}{2}\right)\right) \Gamma\left(\frac{3}{2}-\lambda\right)}{\Gamma\left(\frac{1}{2}\left(\nu-\lambda-\rho+\frac{5}{2}\right)\right) \Gamma\left(\frac{1}{2}\left(\nu+\rho-\lambda+\frac{5}{2}\right)\right) \Gamma\left(\frac{1}{2}\left(\rho-\nu-\lambda+\frac{5}{2}\right)\right)}, \\
\int_{0}^{\infty} \frac{J_{\nu}(b x) J_{\rho}(c x)}{x^{\frac{3}{2}-\lambda}} d x= & \frac{2^{\lambda-\frac{3}{2}} b^{\nu} \Gamma\left(\frac{1}{2}\left(\lambda+\nu+\rho-\frac{1}{2}\right)\right)}{c^{\lambda+\nu-\frac{1}{2}} \Gamma(\nu+1) \Gamma\left(\frac{1}{2}\left(\rho-\nu-\lambda+\frac{5}{2}\right)\right)} \times \\
& F\left(\frac{1}{2}\left(\lambda+\nu+\rho-\frac{1}{2}\right), \frac{1}{2}\left(\lambda+\nu-\rho-\frac{1}{2}\right) ; \nu+1,\left(\frac{b}{c}\right)^{2}\right)
\end{aligned}
$$

which are valid for $c \geq b>0$ at least if $1 / 2-\nu-\rho<\lambda<3 / 2$. These are needed in the proof of Proposition 3.1. To get (A.13), we set $\mu=-1 / 2$ in (A.8) and then let $a \rightarrow 0+$, which is licit by the dominated convergence theorem; (A.12) is then obtained from (A.13) by letting $c \rightarrow b+$ and using the integral representation of $F$ given in (A.6).

\section{Aknowledgements}

We are grateful to Esko Valkeila for useful discussions and for sending us several references.

\section{References}

Bailey, W. N. (1934). Some infinite integrals involving Bessel functions. Proc. Lond. Math. Soc., $40(2), 37-48$.

Barton, R., and Poor, H. (1988). Signal detection in fractional Gaussian noise. IEEE Trans. inf. theory, 34, 943-959. 
Decreusefond, W. N., and Üstünel, A. (1999). Stochastic analysis of the fractional Brownian motion. Potential Analysis, 10, 177-214.

Feyel, D., and de La Pradelle, A. (1996). Fractional integrals and Brownian processes. (Preprint, Université d'Évry)

Gradshteyn, I. S., and Rizhik, I. M. (1980). Tables of integrals, series and products (corrected and enlarged ed.). Academic Press.

Hunt, G. A. (1951). Random Fourier transforms. Trans. Amer. Math. Soc., 71, 38-69.

Kolmogorov, A. N. (1940). Wienersche spiralen und einige andrer interessante kurven im hilbertschen raum. C. R. Doklady Acad. Sci. URSS (N.S.) , 26, 115-118.

Lebedev, N. N. (1972). Special functions and their applications. Dover.

Lipster, R. S., and Shiryayev, A. N. (1989). Theory of martingales. Kluwer.

Mandelbrot, B. B., and van Ness, J. W. (1968). Fractional Brownian motions, fractional noises and applications. SIAM Review(4), 422-437.

Molchan, G. (1969). Gaussian processes with spectra which are asymptotically equivalent to a power of $\lambda$. Theory of probability and its applications, 14, 530-532.

Molchan, G., and Golosov, J. (1969). Gaussian stationary processes with asymptotic power spectrum. Soviet Math. Dokl., 10(1).

Norros, I., Valkeila, E., and Virtamo, J. (1999). An elementary approach to a Girsanov formula and other analytical results on fractional Brownian motions. Bernoulli, 5, 571-587.

Nuzman, C. J., and Poor, V. H. (2000). Linear estimation of self-similar processes via Lamperti's transformation. Journal of Applied Probability, 37(2), 429-452.

Pipiras, V., and Taqqu, M. (2000). Integration questions related to fractional Brownian motion. Probability Theory and Related Fields(118), 251-291.

Samko, S. G., Kilbas, A. A., and Marichev, O. I. (1993). Fractional integrals and derivatives: Theory and applications. Gordon and Breach.

Samorodnitsky, G., and Taqqu, M. (1994). Stable non-Gaussian random processes: Stochastic models with infinite variance. New York: Chapman and Hall.

Watson, G. N. (1944). Theory of Bessel functions (2 ed.). Cambridge University Press.

Yaglom, A. M. (1987). Correlation theory of stationary and related random functions (Vol. 1). Springer-Verlag. 